\title{
Rigorous Simulations of Microwave Scattering From Finite Conductivity Two-Dimensional Sea Surfaces at Low Grazing Angles
}

\author{
David Miret, Gabriel Soriano, and Marc Saillard
}

\begin{abstract}
We present a boundary integral method for the solution of the rigorous problem of microwave scattering from finite conductivity sea surfaces under grazing illumination. Following the locally perturbated plane approach, the roughness is flattened at the edges of a finite patch, allowing us to use a plane wave as incident field. Both theoretical formulation and numerical implementation are addressed. We present simulations of full-polarization radar cross-sectional diagrams for 2-D ocean-like surfaces in both bistatic and monostatic configurations. The conductivity of the sea water is taken into account with a curved surface-impedance approximation, and results are compared with a perfectly conducting surface model. Simulations are finally confronted to approximate theories and empirical sea backscatter models for low grazing angles at L-band, vertical polarization, and a wind speed of $3 \mathrm{~m} / \mathrm{s}$.
\end{abstract}

Index Terms-Computational modelling, integral equations, method of moments, radar cross-sections, sea surface.

\section{INTRODUCTION}

$\mathbf{T}$ HE complexity of the sea surface, together with scattering effects such as multiple reflexions and shadowing, makes it difficult to understand and quantify the wave-surface interactions at low grazing angles of illumination and detection. This difficulty is particularly pronounced at microwave frequencies where the electromagnetic wavelength becomes resonant with the surface roughness. This is one of the reasons why empirical sea clutter models are of common use to interpret the return of many shipborne or coastal radars [1]-[3].

Recently, many electromagnetic wave scattering theories have been developed for or applied to the solution of microwave scattering from 2-D sea surfaces. However, on one hand, approximate models [4]-[7] are restricted to nongrazing configurations, since these first- or second-order methods cannot cope

Manuscript received November 14, 2012; revised March 19, 2013 and June 21, 2013; accepted June 22, 2013. Date of publication July 22, 2013; date of current version March 3, 2014. The Ph.D. grant of D. Miret was supported by DCNS

D. Miret is with DCNS, 83000 Toulon, France, with the Aix-Marseille Université, CNRS/INSU, IRD, MIO, UM 110, 13288 Marseille, France, and also with the Université de Toulon, CNRS/INSU, IRD, MIO, UM 110, 83957 La Garde, France.

G. Soriano is with Aix Marseille Université, National Center for Scientific Research, École Centrale Marseille, Institut Fresnel, 13013 Marseille, France.

M. Saillard is with Aix-Marseille Université, CNRS/INSU, IRD, MIO, UM 110, 13288 Marseille, France, and also with the Université de Toulon, CNRS/INSU, IRD, MIO, UM 110, 83957 La Garde, France.

Color versions of one or more of the figures in this paper are available online at http://ieeexplore.ieee.org.

Digital Object Identifier 10.1109/TGRS.2013.2271384 with shadowing. On the other hand, classical rigorous solutions that modelize multiple scattering [8], [9] require the surface to be illuminated by a tapered beam in order to avoid edge effects. As a direct consequence, the incidence angle is limited by the size of the beam footprint [10]. To reach grazing, some authors have changed the boundary conditions on the surface: either they are made periodic [11], or resistive loadings are added near the edge of the numerical surface [12].

An alternative numerical approach where the boundary conditions are left untouched has been proposed by some of the authors in [13]. In the cited paper, it is described how edge effects can be avoided, whereas the incident field consists in a polarized plane wave. The roughness of the surface no longer covers the full surface sample but is flattened on the edge. With this locally perturbated plane approach, the field in air is the sum of the incident plane wave, i.e., the specularly reflected plane wave and the scattered field. This last field, which shows a spherical wave structure at far field, bears the contribution of the roughness to the total field and only that contribution. A boundary integral formalism has been developed on the tangential components of the so-defined scattered field.

This formalism first leads to universal theoretical results on the behavior of the scattered field by rough surfaces and the associated radar cross section that generalize [14] to electromagnetic waves and natural media. Second, the boundary integral equations have been numerically implemented and solved following a method of moments, which has been named grazing method of moments (GMoM) for the nonce. This implementation on 2-D surfaces was, however, restricted to perfectly conducting surfaces, and applied to simulate only the horizontal component of the scattered field by rough surface in the case of horizontally polarized incident plane wave $(\mathrm{HH}$ component).

The present paper presents the extension of the GMoM to finite conductivity 2-D surfaces in order to simulate microwave sea surface full-polarization radar cross sections at low grazing incidence and detection angles. This paper is organized as follows. In Section II, the surface electromagnetic scattering theory is developed for a locally perturbed plane interfacing two homogeneous media. Then, numerical implementation is detailed in Section III. Sea water is modelized with a curved surface-impedance approximation. The right-hand side of the surface integral equation as well as the scattering formula for the equivalent currents are given complete expressions. In Section IV, numerical results of both bistatic and monostatic 


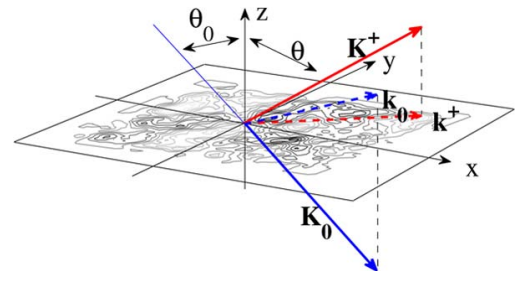

Fig. 1. Geometry of the wave scattering from locally perturbated plane problem.

radar cross sections are presented. Simulations on the first random rough surface, with truncated power-law spectrum and finite conductivity, are the opportunity to determine the accuracy of the extended GMoM through numerical tests. The GMoM is then applied at L-band microwave frequency to a more realistic sea spectrum, at grazing angles in vertical polarization and backscattering configuration. It is compared with two classical surface scattering approximate theories, and confronted to experimental sea clutter data and empirical model. This paper is finally concluded.

\section{BOUNDARY INTEGRAL FORMALISM}

The time-harmonic scattering of a plane wave from the finite conductivity sea surface is considered in the framework of the locally perturbed plane approach. The interface between air and sea water is assumed infinite: in the right Cartesian coordinate $(\hat{\mathbf{x}}, \hat{\mathbf{y}}, \hat{\mathbf{z}})$, the surface is given by equation $z=h(\mathbf{r})=h(x, y)$, with roughness set to zero outside a bounded region of the $(x O y)$ plane (see Fig. 1). Electric and magnetic fields are denoted by $\mathbf{E}$ and $\mathbf{H}$, respectively, with an assumed time dependence $e^{-i \omega t}$ at working frequency $\omega$.

For a given incident field $\left(\mathbf{E}^{i}, \mathbf{H}^{i}\right)$, we first define the reflected field $\left(\mathbf{E}^{r}, \mathbf{H}^{r}\right)$ as the field that would be reflected by the $(x O y)$ plane, that is, the flat sea. Both fields are polarized plane waves. Then, in this paper, we choose to define the scattered field in the air by

$$
\mathbf{E}^{s}=\mathbf{E}-\mathbf{E}^{i}-\mathbf{E}^{r} \quad \mathbf{H}^{s}=\mathbf{H}-\mathbf{H}^{i}-\mathbf{H}^{r}
$$

with $(\mathbf{E}, \mathbf{H})$ denoting the total field. As a consequence, that scattered field at point $\mathbf{R}=\mathbf{r}+z \hat{\mathbf{z}}$ shows a surface or boundary integral representation [15], i.e.,

$$
\begin{aligned}
& -\frac{i}{\omega \varepsilon_{0}} \operatorname{curl}_{\mathbf{R}} \operatorname{curl}_{\mathbf{R}} \int G_{0 \mathbf{R}, \mathbf{R}^{\prime}} \mathbf{m}_{\mathbf{R}^{\prime}} d \Sigma^{\prime} \\
& -\operatorname{curl}_{\mathbf{R}} \int G_{0 \mathbf{R}, \mathbf{R}^{\prime}} \mathbf{j}_{\mathbf{R}^{\prime}} d \Sigma^{\prime}= \begin{cases}\mathbf{E}_{\mathbf{R}}^{s} & \text { if } z>h(\mathbf{r}) \\
\mathbf{0} & \text { if } z<h(\mathbf{r})\end{cases} \\
& +\frac{i}{\omega \mu_{0}} \operatorname{curl}_{\mathbf{R}} \operatorname{curl}_{\mathbf{R}} \int G_{0 \mathbf{R}, \mathbf{R}^{\prime}} \mathbf{m}_{\mathbf{R}^{\prime}} d \Sigma^{\prime} \\
& -\operatorname{curl}_{\mathbf{R}} \int G_{0 \mathbf{R}, \mathbf{R}^{\prime}} \mathbf{j}_{\mathbf{R}^{\prime}} d \Sigma^{\prime}= \begin{cases}\mathbf{H}_{\mathbf{R}}^{s} & \text { if } z>h(\mathbf{r}) \\
\mathbf{0} & \text { if } z<h(\mathbf{r})\end{cases}
\end{aligned}
$$

built upon its tangential components on the surface, i.e.,

$$
\mathbf{m}=\hat{\mathbf{n}} \times \mathbf{E}^{s}, \quad \mathbf{j}=\hat{\mathbf{n}} \times \mathbf{H}^{s}
$$

and the free-space Green's function in the air $G_{0 \mathbf{R}, \mathbf{R}^{\prime}}=$ $-\left(e^{i K_{0}\left|\mathbf{R}-\mathbf{R}^{\prime}\right|}\right) /\left(4 \pi\left|\mathbf{R}-\mathbf{R}^{\prime}\right|\right) . \quad K_{0}=\sqrt{\epsilon_{0} \mu_{0}} \omega$ denotes the wavenumber in the air of permittivity $\varepsilon_{0}$ and permeability $\mu_{0}$. Both the $z$-axis and $\hat{\mathbf{n}}$ the surface unit normal vector are directed toward air. In (2) and (3), the two tangential components $\mathbf{m}$ and $\mathbf{j}$ act as equivalent surface currents. They radiate through the Green's function four vector potentials, i.e., the two fields $\mathbf{E}^{s}$ and $\mathbf{H}^{s}$ writing as combinations of those potentials at any point $z>h(\mathbf{r})$ of the air.

Tangential components of (2) and (3) turn to two boundary integral equations at the limit $z \rightarrow h(\mathbf{r})$ when the observation point $\mathbf{R}$ tends toward the surface, i.e.,

$$
\begin{aligned}
& \left(\frac{1}{2}+M_{0}\right) \mathbf{m}+\frac{i}{\omega \varepsilon_{0}} P_{0} \mathbf{j}=\mathbf{0} \\
& \left(\frac{1}{2}+M_{0}\right) \mathbf{j}-\frac{i}{\omega \mu_{0}} P_{0} \mathbf{m}=\mathbf{0} .
\end{aligned}
$$

Here, it is convenient to introduce the two, namely, magnetic field $M_{0}$ and electric field $P_{0}$ integral operators of the literature [16]. They write for $\mathbf{c}$ a surface current as

$$
\begin{aligned}
M_{0} \mathbf{c}_{\mathbf{R}}=\hat{\mathbf{n}}_{\mathbf{R}} \times \int & \operatorname{grad}_{\mathbf{R}} G_{0 \mathbf{R}, \mathbf{R}^{\prime}} \times \mathbf{c}_{\mathbf{R}^{\prime}} d \Sigma^{\prime} \\
P_{0} \mathbf{c}_{\mathbf{R}}=\hat{\mathbf{n}}_{\mathbf{R}} \times \int( & K_{0}^{2} G_{0 \mathbf{R}, \mathbf{R}^{\prime}} \mathbf{c}_{\mathbf{R}} \\
& \left.\quad+\operatorname{grad}_{\mathbf{R}} G_{0 \mathbf{R}, \mathbf{R}^{\prime}} \operatorname{Div}_{\mathbf{R}^{\prime}}\right) d \Sigma^{\prime} .
\end{aligned}
$$

Now $\mathbf{R}$, just as $\mathbf{R}^{\prime}$, lies on the surface. The surface divergence Div involved in the expression of $P_{0}$ will be given further details in Section III. Equations (5) and (6) constitute two modified integral equations that express with the classical operators.

Equations (5) and (6) being equivalent, a supplementary equation, namely, the boundary condition on the surface $z=$ $h(\mathbf{r})$ is required to solve the scattering problem. Here, the derivation is classical. Assuming that sea water behaves as a semi-infinite homogeneous lossy dielectric with complex relative permittivity $\varepsilon_{r}$ at frequency $\omega$, the tangential components of the total field $\hat{\mathbf{n}} \times \mathbf{E}$ and $\hat{\mathbf{n}} \times \mathbf{H}$ are bounded by the two integral equations, i.e.,

$$
\begin{gathered}
\left(\frac{1}{2}-M\right) \hat{\mathbf{n}} \times \mathbf{E}-\frac{i}{\omega \varepsilon_{0} \varepsilon_{r}} P \hat{\mathbf{n}} \times \mathbf{H}=\mathbf{0} \\
\left(\frac{1}{2}-M\right) \hat{\mathbf{n}} \times \mathbf{H}+\frac{i}{\omega \mu_{0}} P \hat{\mathbf{n}} \times \mathbf{E}=\mathbf{0} .
\end{gathered}
$$

Operators $M$ and $P$ for the water follow expressions similar to (7) and (8), with the permittivity $\varepsilon_{0} \varepsilon_{r}$ in place of that of vacuum. Wavenumber is thus $K_{0} \sqrt{\epsilon_{r}}$, in particular, in the freespace Green's function. From (9), we immediately get

$$
\hat{\mathbf{n}} \times \mathbf{E}=Z \hat{\mathbf{n}} \times \mathbf{H} \quad Z=\frac{i}{\omega \varepsilon_{0} \varepsilon_{r}}\left(\frac{1}{2}-M\right)^{-1} P
$$

and introduce operator $Z$ for sea water. This relation allows us to write the unknown $\mathbf{m}$ as a function of $\mathbf{j}$.

Denoting $\mathbf{m}^{e}=\hat{\mathbf{n}} \times\left(\mathbf{E}^{\mathbf{i}}+\mathbf{E}^{\mathbf{r}}\right)$ and $\mathbf{j}^{e}=\hat{\mathbf{n}} \times\left(\mathbf{H}^{\mathbf{i}}+\mathbf{H}^{\mathbf{r}}\right)$, and in order to get equations on unknowns $\mathbf{m}$ and $\mathbf{j}$, we simply 
use $\mathbf{m}=\hat{\mathbf{n}} \times \mathbf{E}-\mathbf{m}^{e}$ and $\mathbf{j}=\hat{\mathbf{n}} \times \mathbf{H}-\mathbf{j}^{e}$ in (10) and (11). Combining the resulting equations and (6) and resorting to integral operator products properties [17], i.e.,

$$
M P+P M=0, \quad \frac{1}{4}-M^{2}=\frac{P^{2}}{k^{2}}
$$

we get a single nonsingular integral equation

$$
\left(1+M_{0}-M+\frac{P_{0}-P}{i \omega \mu_{0}} Z\right) \mathbf{j}=\frac{-i P_{0}}{\omega \mu_{0}}\left(\mathbf{m}^{e}-Z \mathbf{j}^{e}\right)
$$

that can be numerically solved with a classical method of moments. A key point is that edge effects can be avoided even for a plane wave illumination since the term $\mathbf{m}^{e}-Z \mathbf{j}^{e}$ of the right-hand side vanishes outside the rough region. This point will be illustrated further in Section III.

In this paper, we are interested in the determination of the sea surface scattering in far field conditions. The scattered field as defined in (1) showing a spherical wave structure, (2) leads to the limit

$$
\mathbf{E}_{\mathbf{R}}^{s} \sim-i \frac{e^{i K_{0} R}}{R} \mathbf{S}^{+}(\mathbf{k}), \quad \mathbf{S}^{-}(\mathbf{k})=\mathbf{0} .
$$

$\mathbf{K}^{+}$and $\mathbf{K}^{-}$correspond to upward- and downward-directed wave vectors, respectively, with horizontal component $\mathrm{k}$ and vertical component $\pm q$, i.e.,

$$
\mathbf{K}^{ \pm}=K_{0} \frac{\mathbf{R}}{R}=\mathbf{k} \pm q \hat{\mathbf{z}} .
$$

The vector scattering amplitude $\mathbf{S}^{ \pm}(\mathbf{k})$, i.e.,

$$
\begin{array}{r}
\mathbf{S}^{ \pm}(\mathbf{k})=\frac{1}{4 \pi} \int\left(-\mathbf{K}^{ \pm} \times \mathbf{m}_{\mathbf{R}}+\frac{1}{\omega \varepsilon_{0}} \mathbf{K}^{ \pm} \times\left(\mathbf{K}^{ \pm} \times \mathbf{j}_{\mathbf{R}}\right)\right) \\
\cdot \exp \left(-i \mathbf{K}^{ \pm} \cdot \mathbf{R}\right) d \Sigma
\end{array}
$$

is denoted $\mathbf{S}_{\alpha}^{ \pm}(\mathbf{k})$ when the incident field is a plane wave $\mathbf{E}_{\mathbf{R}}^{i}=e^{i\left(\mathbf{k}_{0} \cdot \mathbf{r}-q_{0} z\right)} \hat{\boldsymbol{\alpha}}$ of unit complex amplitude, wave vector $\mathbf{K}_{0}=\mathbf{k}_{0}-q_{0} \hat{\mathbf{z}}$, and vertical $\hat{\boldsymbol{\alpha}}=\hat{\mathbf{v}}_{0}=k_{0} \hat{\mathbf{z}}+q_{0} \hat{\mathbf{k}}_{0}(\alpha=V)$ or horizontal $\hat{\boldsymbol{\alpha}}=\hat{\mathbf{h}}_{0}=\hat{\mathbf{k}}_{0} \times \hat{\mathbf{z}}(\alpha=H)$ linear polarization. Normal to wave vector $\mathbf{K}^{ \pm}$, it decomposes as $\mathbf{S}_{\alpha}^{ \pm}=S_{V \alpha}^{ \pm} \hat{\mathbf{v}}^{ \pm}+$ $S_{H \alpha}^{ \pm} \hat{\mathbf{h}}$ onto the basis of vertical $(\beta=V) \hat{\mathbf{v}}^{ \pm}=k \hat{\mathbf{z}} \mp q \hat{\mathbf{k}}$ and horizontal $(\beta=H) \hat{\mathbf{h}}=\hat{\mathbf{k}} \times \hat{\mathbf{z}}$ linear polarizations with components, i.e.,

$$
\begin{aligned}
& S_{V \alpha}^{ \pm}(\mathbf{k})=-\frac{K_{0}}{4 \pi} \int\left(\hat{\mathbf{v}}^{ \pm} \cdot \eta_{0} \mathbf{j}_{\mathbf{R}}-\hat{\mathbf{h}} \cdot \mathbf{m}_{\mathbf{R}}\right) e^{-i \mathbf{K}^{ \pm} \cdot \mathbf{R}} d \Sigma \\
& S_{H \alpha}^{ \pm}(\mathbf{k})=-\frac{K_{0}}{4 \pi} \int\left(\hat{\mathbf{v}}^{ \pm} \cdot \mathbf{m}_{\mathbf{R}}+\hat{\mathbf{h}} \cdot \eta_{0} \mathbf{j}_{\mathbf{R}}\right) e^{-i \mathbf{K}^{ \pm} \cdot \mathbf{R}} d \Sigma
\end{aligned}
$$

where $\eta_{0}=\sqrt{\mu_{0} / \varepsilon_{0}}$ is the electrical impedance of air. The $S_{\beta \alpha}^{+}(\mathbf{k})$ are the four coefficients of the scattering matrix.

Since $\mathbf{E}^{s}$ and the standard scattered field defined as $\mathbf{E}-$ $\mathbf{E}^{i}$ share the same incoherent component, the microwave sea surface incoherent normalized radar cross section (NRCS) is related to the scattering amplitude by

$$
\sigma_{\beta \alpha}^{0}\left(\mathbf{k}, \mathbf{k}_{0}\right)=\frac{4 \pi}{A}\left\langle\left|S_{\beta \alpha}^{+}(\mathbf{k})-\left\langle S_{\beta \alpha}^{+}(\mathbf{k})\right\rangle\right|^{2}\right\rangle
$$

with $A$ standing here for the area of the rough region.

For propagative waves, the vertical components of the incident and scattering wave vectors are simply related to the incident and scattering angles, i.e.,

$$
q_{0}=K_{0} \cos \theta_{0}, \quad q=K_{0} \cos \theta .
$$

As discussed in [13], the behavior of the NRCS at grazing incidence and scattering angles is universal for finite conductivity surfaces in that sense that it does not depend on the roughness or permittivity: using the Landau notation, it writes

$$
\sigma_{\beta \alpha}^{0}\left(\mathbf{k}, \mathbf{k}_{0}\right)=O\left(q_{0}^{2} q^{2}\right) .
$$

For a perfectly conducting surface, this behavior is only met by the HH component of the NRCS.

\section{Numerical IMPLEMENTATION}

Following the model introduced in [13], let us consider a rough surface, with roughness flattened except on a finite area, whose size is chosen to ensure that the right-hand size of (13) is bounded to the numerical domain, with a smooth transition between the flat part and the rough region.

In the case of strongly conductive surfaces, such as sea surface or very wet soils at microwave frequencies, or metals in optics, an impedance boundary condition is relevant. With a skin depth much smaller than the electromagnetic wavelength in the air $\lambda=2 \pi / K_{0}$, interactions in the sea water are restricted to the shorter range, and $Z$ can be accurately approximated by a local operator. Depending only on the sea water complex skin depth $d=i / K$ and on the geometrical features of the surface, its expression is given in [18]. A tangential vector c satisfies $\hat{\mathbf{n}} \cdot \mathbf{c}=\mathbf{0}$ with $\phi \hat{\mathbf{n}}=-(\partial h / \partial x) \hat{\mathbf{x}}-(\partial h / \partial y) \hat{\mathbf{y}}+\hat{\mathbf{z}}$ and $\phi=\sqrt{1+(\partial h / \partial x)^{2}+(\partial h / \partial y)^{2}}$; therefore, its Cartesian coordinates are related by $c_{z}=(\partial h / \partial x) c_{x}+(\partial h / \partial y) c_{y}$. Surface vector operators, be they local as $Z$ or integral as $M_{0}$ and $P_{0}$, apply to and output tangential vectors. As such, they can be represented by $2 \times 2$ matrices. Relating the two first Cartesian coordinates of tangential vectors $\hat{\mathbf{n}} \times \mathbf{E}$ and $\hat{\mathbf{n}} \times \mathbf{H}$, the boundary condition $\hat{\mathbf{n}} \times \mathbf{E}=Z \hat{\mathbf{n}} \times \mathbf{H}$ writes

$$
\left[\begin{array}{l}
\hat{\mathbf{x}} \cdot(\hat{\mathbf{n}} \times \mathbf{E}) \\
\hat{\mathbf{y}} \cdot(\hat{\mathbf{n}} \times \mathbf{E})
\end{array}\right]=\left[\begin{array}{ll}
Z_{11} & Z_{12} \\
Z_{21} & Z_{22}
\end{array}\right]\left[\begin{array}{l}
\hat{\mathbf{x}} \cdot(\hat{\mathbf{n}} \times \mathbf{H}) \\
\hat{\mathbf{y}} \cdot(\hat{\mathbf{n}} \times \mathbf{H})
\end{array}\right]
$$

with the surface impedance [18], i.e.,

$$
\begin{aligned}
{\left[\begin{array}{ll}
Z_{11} & Z_{12} \\
Z_{21} & Z_{22}
\end{array}\right]=} & \frac{\eta_{0}}{\phi \sqrt{\varepsilon_{r}}}\left[\begin{array}{cc}
0 & -1 \\
+1 & 0
\end{array}\right] \\
& \cdot\left\{\left(1-\frac{i J}{2 K_{0} \sqrt{\varepsilon_{r}}}\right)\left[\begin{array}{cc}
1+\left(\frac{\partial h}{\partial x}\right)^{2} & \frac{\partial h}{\partial x} \frac{\partial h}{\partial y} \\
\frac{\partial h}{\partial x} \frac{\partial h}{\partial y} & 1+\left(\frac{\partial h}{\partial y}\right)^{2}
\end{array}\right]\right. \\
& \left.+\frac{i}{K_{0} \sqrt{\varepsilon_{r}}}\left[\begin{array}{cc}
\frac{\partial^{2} h}{\partial x^{2}} & \frac{\partial^{2} h}{\partial x \partial y} \\
\frac{\partial^{2} h}{\partial x \partial y} & \frac{\partial^{2} h}{\partial y^{2}}
\end{array}\right]\right\}
\end{aligned}
$$


and mean curvature

$$
\begin{aligned}
J=\frac{1}{\phi^{3}}\left\{\left(1+\left(\frac{\partial h}{\partial y}\right)^{2}\right)\right. & \frac{\partial^{2} h}{\partial x^{2}}-2 \frac{\partial h}{\partial x} \frac{\partial h}{\partial y} \frac{\partial^{2} h}{\partial x \partial y} \\
+ & \left.\left(1+\left(\frac{\partial h}{\partial x}\right)^{2}\right) \frac{\partial^{2} h}{\partial y^{2}}\right\} .
\end{aligned}
$$

Note that with such a boundary condition, the reflexion coefficients at incidence angle $\theta_{0}$ slightly differ from the Fresnel's

$$
r_{H}=\frac{\cos \theta_{0}-\sqrt{\epsilon_{r}}}{\cos \theta_{0}+\sqrt{\epsilon_{r}}} \quad r_{V}=\frac{\epsilon_{r} \cos \theta_{0}-\sqrt{\epsilon_{r}}}{\epsilon_{r} \cos \theta_{0}+\sqrt{\epsilon_{r}}} .
$$

Those coefficients rule the amplitude of the reflected field $\left(\mathbf{E}^{r}, \mathbf{H}^{r}\right)$ and ensure that term $\mathbf{m}^{e}-Z \mathbf{j}^{e}$ of the right-hand side of our integral equation effectively vanishes outside the rough region. As an illustrative example, let us derive the case of a horizontally polarized plane wave with a wave vector in the $(x O z)$ plane in a point where the surface is flat. The curved surface impedance (21) simplifies for a horizontal plane

$$
\begin{aligned}
{\left[\begin{array}{ll}
Z_{11} & Z_{12} \\
Z_{21} & Z_{22}
\end{array}\right] } & =\frac{\eta_{0}}{\sqrt{\varepsilon_{r}}}\left[\begin{array}{cc}
0 & -1 \\
+1 & 0
\end{array}\right] \\
\hat{\mathbf{z}} \times \mathbf{E} & =\frac{\eta_{0}}{\sqrt{\varepsilon_{r}}} \hat{\mathbf{z}} \times(\hat{\mathbf{z}} \times \mathbf{H})
\end{aligned}
$$

to the so-called Leontovich boundary condition. From Maxwell's equations, we easily find

$$
\begin{aligned}
\mathbf{m}^{e} & =-E_{0} e^{i K_{0} \sin \theta_{0} x}\left(1+r_{H}\right) \hat{\mathbf{x}} \\
\mathbf{j}^{e} & =\frac{-E_{0} K_{0}}{\omega \mu_{0}} \cos \theta_{0}\left(1-r_{H}\right) e^{i K_{0} \sin \theta_{0} x} \hat{\mathbf{y}} \\
Z \mathbf{j}^{e} & =-E_{0} e^{i K_{0} \sin \theta_{0} x} \frac{\cos \theta_{0}}{\sqrt{\epsilon_{r}}}\left(1-r_{H}\right) \hat{\mathbf{x}} .
\end{aligned}
$$

We see that the equation $\mathbf{m}^{e}=Z \mathbf{j}^{e}$ is equivalent to $\sqrt{\epsilon_{r}}(1+$ $\left.r_{H}\right)=\cos \theta_{0}\left(1-r_{H}\right)$, which is true given the expression of the reflexion coefficients (23).

Integral equation (13) is solved using a method of moments [19]. The unknown scalar functions are $j_{x}$ and $j_{y}$, since $j_{z}=$ $(\partial h / \partial x) j_{x}+(\partial h / \partial y) j_{y}$. The vector equation is projected onto the $\hat{\mathbf{x}}$ and $\hat{\mathbf{y}}$ axes to obtain an integral system of two coupled scalar equations. The rough surface is parametrized by coordinates $x$ and $y$. With the elementary surface writing $d \Sigma=$ $\phi d x d y$, surface integrals are turned into double integrals, and then bounded to a rectangular numerical domain of $\mathbb{R}^{2}$ that encompasses the rough region and the assumed support of the unknown functions. We now discretize. The numerical domain is regularly meshed with square elements. Functions $\phi j_{x}$ and $\phi j_{y}$ (rather than $j_{x}$ and $j_{y}$, this is common practice [20, p. 281]) are expanded on the piecewise-constant basis functions of the elements. With point-matching technique, equations are tested at the center of each element, to get a linear system $A X=B$ with a completely filled matrix $A$. $N$ being the number of elements, this system of order $2 N$ can be solved iteratively at a $O\left(N^{2}\right)$ cost in central memory and computing time. For 2-D surfaces, $N$ becomes very large; thus, advanced numerical schemes have been proposed. We use a multilevel canonical grid technique [21]; memory use and time cost are reduced to $O(N)$ and $O(N \log N)$, respectively; details of the implementation can be found in [9].

Operators $M_{0}, M$, and $P_{0}-P$ in the left-hand side of (13) being weakly singular, they can be safely discretized with piecewise-constant basis functions and point matching. On the contrary, operator $P_{0}$ is hypersingular and involves a Cauchy principal value surface integral over the surface divergence of the current (8). The computation of the right-hand side of (13) is thus nontrivial and is now detailed.

The surface divergence of a surface vector density $\mathbf{c}(x, y)$ can be defined as follows: if $\mathbf{c}$ is the tangential component of some vector field $\mathbf{A}$, then [22], i.e.,

$$
\begin{aligned}
\mathbf{c}(x, y) & =\hat{\mathbf{n}} \times \mathbf{A}(x, y, h(x, y)) \\
\operatorname{Div} \mathbf{c} & =-\hat{\mathbf{n}} \cdot \operatorname{curl} \mathbf{A}=\frac{1}{\phi}\left(\frac{\partial\left(\phi c_{x}\right)}{\partial x}+\frac{\partial\left(\phi c_{y}\right)}{\partial y}\right) .
\end{aligned}
$$

We thus develop

$$
\begin{aligned}
\operatorname{Div}\left(\mathbf{m}^{e}-Z \mathbf{j}^{e}\right) & =\operatorname{Div} \mathbf{m}^{e}-\operatorname{Div}\left(Z \mathbf{j}^{e}\right) \\
\operatorname{Div} \mathbf{m}^{e} & =-\hat{\mathbf{n}} \cdot \operatorname{curl}\left(\mathbf{E}^{i}+\mathbf{E}^{r}\right) \\
& =-i \omega \mu_{0} \hat{\mathbf{n}} \cdot\left(\mathbf{H}^{i}+\mathbf{H}^{r}\right)=-i \omega \mu_{0} H_{n}^{e} \\
\operatorname{Div}\left(Z \mathbf{j}^{e}\right)= & \frac{1}{\phi}\left(\frac{\partial}{\partial x}\left[Z_{11} \phi j_{x}^{e}+Z_{12} \phi j_{y}^{e}\right]\right. \\
& \left.\quad+\frac{\partial}{\partial y}\left[Z_{21} \phi j_{x}^{e}+Z_{22} \phi j_{y}^{e}\right]\right) \\
& \simeq \frac{1}{\phi}\left(Z_{11} \frac{\partial \phi j_{x}^{e}}{\partial x}+Z_{12} \frac{\partial \phi j_{y}^{e}}{\partial x}\right. \\
& \left.\quad+Z_{21} \frac{\partial \phi j_{x}^{e}}{\partial y}+Z_{22} \frac{\partial \phi j_{y}^{e}}{\partial y}\right) .
\end{aligned}
$$

In the evaluation of (36), it has been numerically shown that the spatial derivatives of the components of $Z$ can be neglected, leading to simplified expression (37).

For the right-hand side $\left(-i / \omega \mu_{0}\right) P_{0}\left(\mathbf{m}^{e}-Z \mathbf{j}^{e}\right)$ to be computed, $H_{n}^{e}$ as well as $j_{x}^{e}, j_{y}^{e}$ and their derivatives with respect to $x$ and $y$ are evaluated analytically for an incident plane wave of given angle and polarization and expanded in piecewise-constant basis functions. The evaluation of the righthand side vector $B$ of the linear system is obtained from two matrix-vector products, therefore requires $O\left(N^{2}\right)$ operations. With the multilevel canonical grid technique anew, it is performed with reduced $O(N)$ memory and $O(N \log N)$ time requirements.

Once the equivalent currents $\mathbf{m}$ and $\mathbf{j}$ are determined for $\mathbf{a}$ given roughness and incident field, the scattered field has to be computed. It has been established in (14) that $S_{\beta \alpha}^{-}(\mathbf{k})=$ 0 whatever direction and polarization; thus, we are allowed to use for numerical calculations the same combination we used for the perfectly conducting case, $H H$-polarized scattered 
field in [13]: $S_{\beta \alpha}^{d i f}(\mathbf{k})=S_{\beta \alpha}^{+}(\mathbf{k})-S_{\beta \alpha}^{-}(\mathbf{k})$. It writes in each polarization case, i.e.,

$$
\begin{aligned}
S_{V \alpha}^{d i f}(\mathbf{k})=\frac{1}{2 \pi} \int\{ & i \sin (q h(\mathbf{r}))\left(k \hat{\mathbf{z}} \cdot \eta_{0} \mathbf{j}_{\mathbf{R}}-K_{0} \hat{\mathbf{h}} \cdot \mathbf{m}_{\mathbf{R}}\right) \\
& \left.+q \cos (q h(\mathbf{r})) \hat{\mathbf{k}} \cdot \eta_{0} \mathbf{j}_{\mathbf{R}}\right\} e^{-i \mathbf{k} \cdot \mathbf{r}} d \Sigma \\
S_{H \alpha}^{d i f}(\mathbf{k})=\frac{1}{2 \pi} \int\{ & i \sin (q h(\mathbf{r}))\left(k \hat{\mathbf{z}} \cdot \mathbf{m}_{\mathbf{R}}+K_{0} \hat{\mathbf{h}} \cdot \eta_{0} \mathbf{j}_{\mathbf{R}}\right) \\
& \left.+q \cos (q h(\mathbf{r})) \hat{\mathbf{k}} \cdot m_{R}\right\} e^{-i \mathbf{k} \cdot \mathbf{r}} d \Sigma .
\end{aligned}
$$

These two expressions, theoretically equivalent to (17)and (18), have the advantage of numerically enforcing the universal behavior (21) of the field and NRCS at grazing scattering angles.

\section{Numerical Results}

Here, we present both bistatic and monostatic simulations of NRCS for 2-D ocean-like surfaces. Our formalism is also tested in two ways, namely, convergence when the number of sampling points per wavelength rises and equality between the two cross-polarized components. Furthermore, small height limit is investigated. Then, the importance of taking into account the finite conductivity of the sea surface at grazing angles is illustrated. Finally, we compare simulations to a pproximate theories, microwave data and a literature empirical model.

The roughness is here statistically characterized by a height spectrum $\Psi\left(\mathbf{k}_{s}\right)$ depending on the surface wave vector $\mathbf{k}_{s}$. The sea surface exhibits a continuous spectrum from millimeter waves to decameter waves and more, depending on wind speed and fetch. For generality purpose, we start with a truncated power-law spectrum surface: the spectral density is set to $\psi\left(\mathbf{k}_{s}\right)=4.10^{-3} k_{s}^{-4}$, where the spatial wavenumber $k_{s}$ goes from $k_{0} / 15$ to $4 k_{0}$. Waves much shortest that the electromagnetic wavelength are disregarded, since it is assumed that they have negligible impact on the scattering process. This spectrum includes the resonant roughness scales, which are important to investigate for radars operating at microwave frequencies. For this surface, the relative complex permittivity is set to $\epsilon_{r}=73.5+61 i$, a typical value for the sea water at L-band $(1.25 \mathrm{GHz})$ obtained with model [23] for a sea surface temperature of $15^{\circ} \mathrm{C}$ and a salinity of $35 \mathrm{psu}$. The discretized surface is square with a $50 \lambda$ side, with $\lambda=24 \mathrm{~cm}$ the radar wavelength. The rough region is $45 \lambda$ large, including $3.75 \lambda$-wide transition zones on each sides. In this paper, all NRCS are estimated by Monte Carlo average over 96 realizations.

We start with bistatic diagrams in the plane of incidence of the VV, HH, HV, and VH NRCS for an incidence angle of $89^{\circ}$, that is, $1^{\circ}$ from grazing. We show in Fig. 2 a very good stability of the results as a function of the number of points per wavelength. Even at most grazing incidence, the scattered field appears not to be modified in any direction when the sampling rate goes from 10 to 15 points per wavelength. This remains true even for the $\mathrm{HH}$ at grazing backward angles and the cross polarizations on the whole diagram, which power is low. This proves that 10 points per wavelength are enough

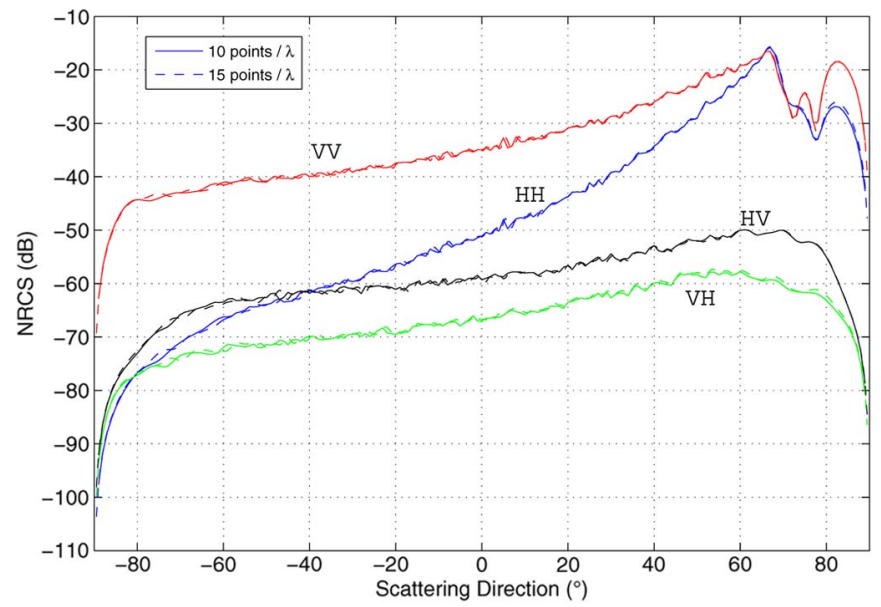

Fig. 2. Simulations of VV, HH, HV, and VH bistatic NRCS at $89^{\circ}$ incidence for a $\left[k_{0} / 15 ; 4 k_{0}\right]$-truncated power-law spectrum 2 -D random rough surface of permittivity $\epsilon_{r}=73.5+61 i$ against scattering angle in the plane of incidence. Two different surface sampling steps are considered for the GMoM, namely, 10 and 15 points per electromagnetic wavelength in the air.

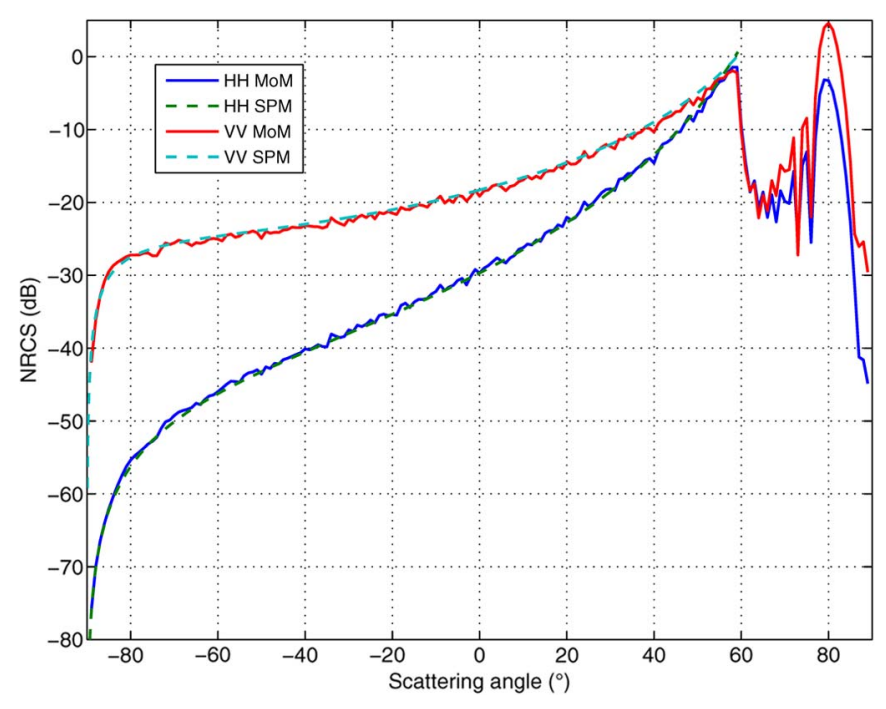

Fig. 3. Simulations of $\mathrm{VV}$ and $\mathrm{HH}$ bistatic NRCS at $80^{\circ}$ incidence for a $\left[k_{0} / 4 ; 4 k_{0}\right]$-truncated power-law spectrum 2 -D random rough surface of permittivity $\epsilon_{r}=73.5+61 i$ against scattering angle in the plane of incidence. The GMoM is compared with the first-order SPM.

for the discretization of such a sea surface scattering problem, even at low grazing angles. Here, we stress the fact that the highest spatial wavenumber in the surface is $4 k_{0}$. This level of discretization, as well as the $4 k_{0}$ high-frequency cutoff, have been kept in every following calculations.

With the low-frequency bound of the spectral density set to $k_{0} / 15$, the surface height root mean square is $h=25.6 \mathrm{~mm}$. Simulations have shown that this value is still too large for GMoM to be compared with the small perturbation method (SPM) [24]. This perturbative development should be retrieved asymptotically at low radar frequency, or for height root mean square much smaller than the radar wavelength. The lowfrequency bound has to be raised up to $k_{0} / 4$ in order to get a satisfying height root mean square of $h=6.8 \mathrm{~mm} \simeq \lambda / 35$. In Fig. 3, the GMoM is compared with the first-order SPM in the same configuration as in Fig. 2. At first-order, the SPM predicts 


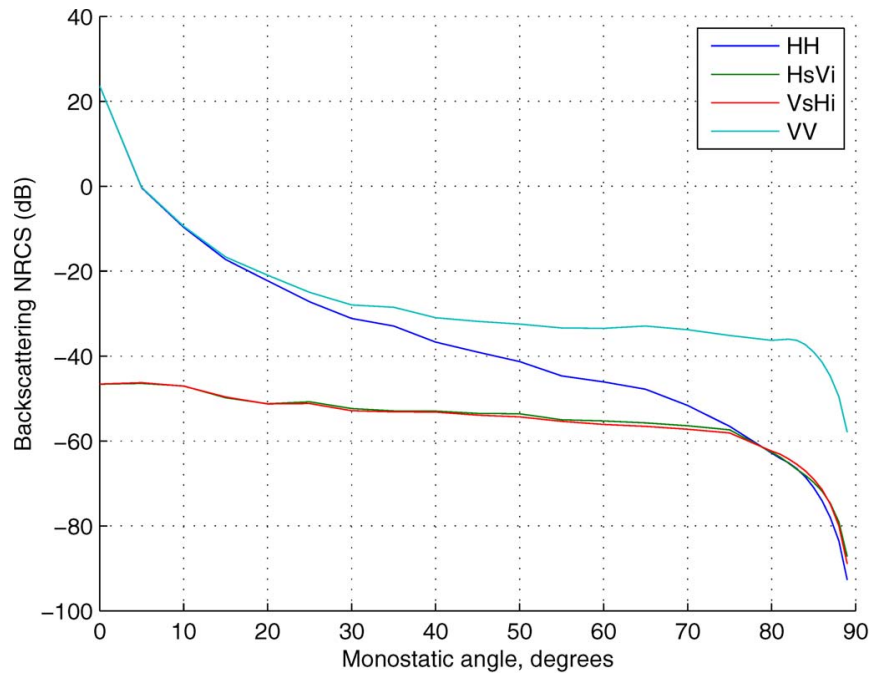

Fig. 4. Simulations of VV, HH, HV, and VH monostatic NRCS for a truncated power-law spectrum 2-D random rough surface of permittivity $\epsilon_{r}=73.5+$ $61 i$ against monostatic angle.

nonzero NRCS only between $-90^{\circ}$ and $60^{\circ}$, but over this range, exact and asymptotic theories perfectly match, within the 1-dB oscillations of the GMoM curve.

We also simulate monostatic diagrams, with an angle step of $5^{\circ}$ from normal to $80^{\circ}$, then $1^{\circ}$ up to $89^{\circ}$, and the last degree is sampled with ten points. The reciprocity theorem, that notably states that in backscattering, both cross-polarized coefficients are equal, is not automatically fulfilled by the method of moments and thus gives us the opportunity to test again our method. Fig. 4 shows that the HV and VH NRCS perfectly coincide at any monostatic angle, even grazing. This is a meaning accuracy test because of the low intensity of the field in cross polarization.

We now illustrate the importance of taking into account the finite conductivity of the sea surface when grazing angles are addressed. For this purpose, we compare the new formalism developed in this paper with the one for perfectly conducting surfaces introduced in [13]. Theory predicts that in HH polarization the cross sections should be very similar, with a $O\left(q_{0}^{4}\right)$ behavior at grazing incidence and backscattering configuration, whereas it is not physical to assume the surface to be perfectly conducting for the case of V-polarized incident field around and beyond the Brewster angle.

The monostatic diagrams of Fig. 5 confirm that conductivity is of negligible influence on the HH NRCS. The difference between the two curves, being around $1.5 \mathrm{~dB}$ at normal and $1 \mathrm{~dB}$ after $45^{\circ}$, is finally smaller than one-tenth $\mathrm{dB}$ after $80^{\circ}$. The opposite behavior is met by the VV NRCS, with a gap of $1.5 \mathrm{~dB}$ at normal, $2 \mathrm{~dB}$ at $60^{\circ}$, and $5 \mathrm{~dB}$ at $80^{\circ}$ and widening at grazing since the perfectly conductivity model predicts a VV NRCS tending toward a finite nonzero limit at grazing. Fig. 5 also shows the perfectly conducting surface is no good model for cross polarizations, with an error always larger than $2 \mathrm{~dB}$ and a NRCS, which does not tend toward zero fast enough.

This formalism has been developed for the study of the sea surface with a microwave radar. The size of the surface needs to be large enough to include the largest waves of the sea surface.

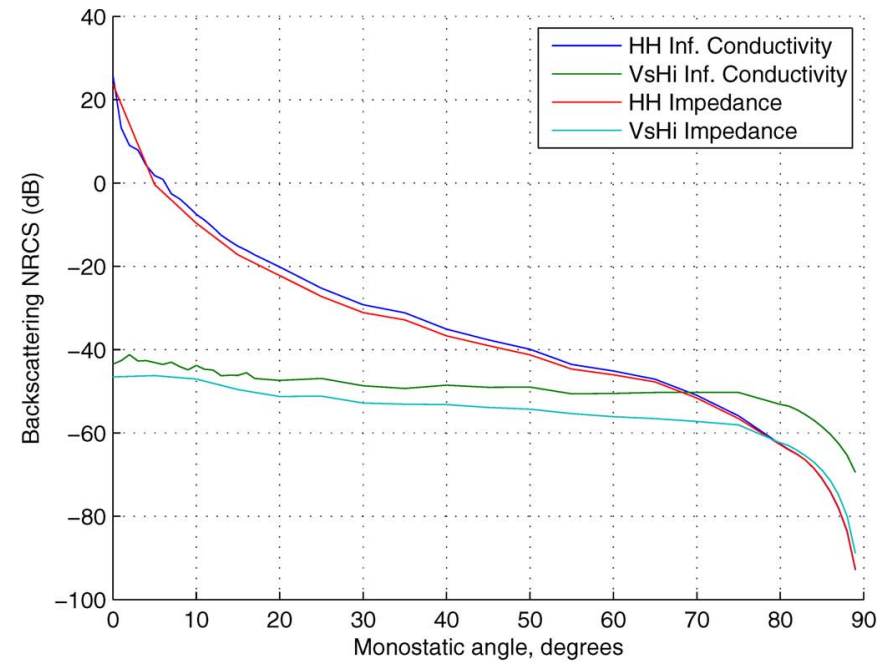

(a)

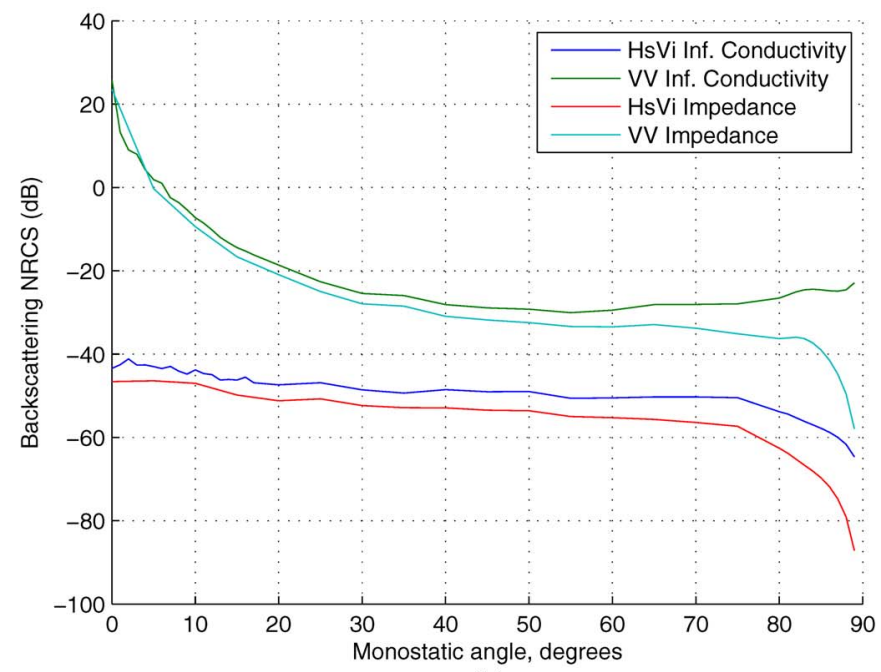

(b)

Fig. 5. Simulations of VV, HH, HV, and VH monostatic NRCS for a truncated power-law spectrum 2-D random rough surface against monostatic angle. The perfectly conducting case and lossy dielectric case with permittivity $\epsilon_{r}=$ $73.5+61 i$ are considered.

Convergence test with the surface size can be performed. The following criterion has been found satisfying: the surfaces has to be at least 2.5 times larger than the gravity peak wavelength. For instance, this peak wavelength for a fully developed winddriven sea is around $8.2 \mathrm{~m}$ if the wind speed is $3 \mathrm{~m} / \mathrm{s}, 22.7 \mathrm{~m}$ if the wind speed is $5 \mathrm{~m} / \mathrm{s}$, and $44.5 \mathrm{~m}$ if the wind speed is $7 \mathrm{~m} / \mathrm{s}$. In the case of the wind speed of $3 \mathrm{~m} / \mathrm{s}$, with a frequency of $1.25 \mathrm{GHz}$, it corresponds to a minimum of 85 electromagnetic wavelengths for the side of the surface.

We present here a first result of comparison between simulations and experimental data, as an example of what this numerical method is capable of. We compute backscattering of a $1.25-\mathrm{GHz}$ plane wave by a sea surface generated using a more realistic height spectrum, i.e., the so-called unified directional spectrum [25] by Elfouhaily et al. The wind speed is set to $3 \mathrm{~m} / \mathrm{s}$, its side is now $100 \lambda$, and dimensions of the rough region and transition zones are also doubled, so that normalizing area is $A=5401.9 \lambda^{2}$. Permittivity is maintained at $\epsilon_{r}=73.5+61 i$. 


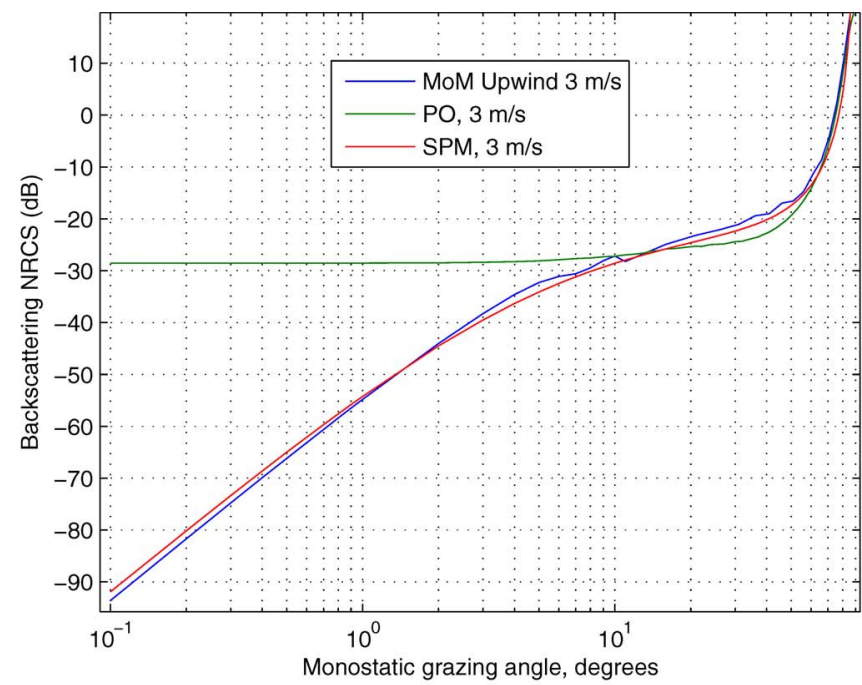

(a)

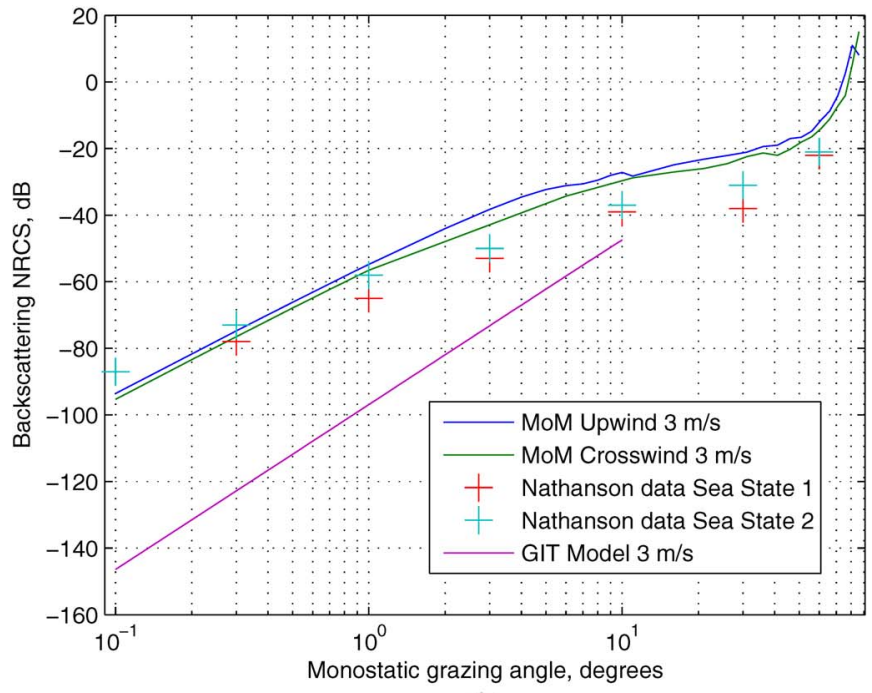

(b)

Fig. 6. GMoM is compared with approximate surface scattering theories (a) and data models (b) for L-band VV monostatic sea surface NRCS at low wind speed against grazing angle.

In Fig. 6, the VV NRCS is plotted against the grazing angle, which is complementary to the incidence angle, so that the most grazing angles are now on the right of the figure. The GMoM is compared with approximate surface scattering theories [26] in Fig. 6(a) and to literature empirical models derived from in situ measurements in Fig. 6(b). We chose two of the most classical approximations, namely, the first-order SPM [24] that corresponds to the low-frequency asymptotics and the physical optics (PO) [27], which is the high-frequency form of the Kirchhoff-tangent plane approximation. Statistical formulations are used for these two methods. The experimental data we use here [1] have been collected by Nathanson and are the results of about 60 experiments performed since 1969 , it is believed that they are the best available data set. Since those data result from measurements averaged over the wind directions, we present backscattering NRCS for the upwind and crosswind configurations in Fig. 6(b), whereas comparisons with SPM and PO in Fig. 6(a) are only upwind. The data are available for different sea states corresponding to different wind speeds, ours $(3 \mathrm{~m} / \mathrm{s})$ is between sea states 1 and 2 .

On one hand, the domain of validity of the PO clearly states that this approximation is not relevant at grazing. On the other hand, SPM is traditionally given to be more accurate at large angles, but its validity at grazing where multiple scattering and shadowing occur is controversial. The GMoM confirms that the PO is to be used around nadir and for monostatic angles smaller than $25^{\circ}$, whereas SPM is perfectly fitting at intermediate angles from $25^{\circ}$ to $70^{\circ}$, only differing from the rigorous solution by $3 \mathrm{~dB}$ at $0.1^{\circ}$ grazing. The GMoM can quantify the error in decibels attached to the use of an approximate surface scattering model, notably at grazing. The remarkable performance of SPM at grazing is now established but restricted to the presently studied configuration, namely, VV polarization and sea surface for a wind speed of $3 \mathrm{~m} / \mathrm{s}$ at L-band. We now consider comparison with data of Fig. 6(b). The agreement between the GMoM and Nathanson data is fair, with the level and shape of the curve retrieved, but the discrepancy can reach $10 \mathrm{~dB}$ between $1^{\circ}$ and $10^{\circ}$ grazing. Since the GMoM is a rigorous method, there is no surface scattering bias. Therefore, variance is explained by three different causes, namely, uncertainties of the experiments, imperfect modelization of the sea surface, and nonsurface scattering phenomena. Nathanson indicates in his book that 5-dB errors are not unlikely. In our simulations, the sea surface samples are obtained by summing independent waves [20]. Interactions between waves, which are predicted by Navier-Stokes equations and lead to breaking waves, are disregarded while they may contribute at grazing. In addition, the surface is assumed to separate sea water from dry air. The humidity that rapidly decreases with height, creating evaporation ducts, is also neglected. The GMoM can also serve for determining the validity of empirical models: as noted in [3], the Georgia Institute of Technology (GIT) model, an empirical model widely used [2], fails to reproduce the behavior of the radar backscattering at grazing incidence for calm seas. This statement is clearly corroborated by our simulations [see Fig. 6(b)].

\section{CONCLUSION}

In this paper, the GMoM have been extended to finite conductivity surfaces and applied to the simulation of 2-D sea surface radar cross section at L-band frequency. Since the incident field is a plane wave, the most grazing angles can be considered. The excellent accuracy of the method at any angle has been emphasized with two numerical tests, namely, convergence with the sampling step and reciprocity theorem, and the small perturbation limit is retrieved for a radar wavelength over surface height root-mean-square ratio of 35 . Comparisons with the perfectly conducting model are also performed, proving the importance of taking into account the finite conductivity of the sea water in the vertical and cross-polarization cases at low grazing angles.

In our simulations, we focus on surface scattering and neglect any other phenomena such as evaporation ducts. In addition, our representation of the sea surface, based on the unified directional sea spectrum, can be improved in a number of ways. 
Nevertheless, in vertical polarization, the case where those defaults have the smallest impact on the radar cross section, our simulations have been fairly compared with Nathanson's sea clutter data of low grazing angles at L-band and sea states 1 and 2. The GIT model has been also assessed in similar conditions.

With the GMoM, we have proven that incidence is no more a limit for the rigorous solution of electromagnetic scattering from 2-D rough surfaces. The numerical burden and, in particular, the computing time, still limits the size of the surface samples. For a frequency of $1.25 \mathrm{GHz}$ and a wind speed of $3 \mathrm{~m} / \mathrm{s}$, the sea patch is 100 electromagnetic wavelength square, requiring around $30 \mathrm{~min}$ of $\mathrm{CPU}$ time on a serial machine for each realization in given geometry, incident angle, and sea conditions. Bistatic diagrams are performed by the Monte Carlo process over 96 realizations. This computing time, which already includes an acceleration technique [9] and the use of optimized mathematical libraries for linear algebra operations and Fourier transforms, roughly varies as the squared frequency and the fourth power of the wind speed! The GMoM can be thus applied to the complete surface spectrum of a fully developed wind-driven sea but only if the wind speed and microwave frequency are sufficiently low. The numerical burden does not allow us at the moment to exceed $7 \mathrm{~m} / \mathrm{s}$ wind speed nor to use frequencies higher than the ones in the C-band. Hardware acceleration, through the use of graphical processing units and associated libraries, should decrease this computing time by one order of magnitude [28]. In addition, up-to-date higher order discretization schemes for the integral equations, such as [29], are compatible with our approach and can be implemented to globally reduce the number of unknowns.

For all that, it should not be concluded that the GMoM for sea surfaces is restricted to low wind speeds and microwave frequencies, since fetch-limited seas can be considered. Our method can be also applied with benefit to truncated sea spectra, since the most difficult and important waves to modelize in scattering are the resonant ones, which are smaller than 15 electromagnetic wavelengths. This methodology has already been followed for 2-D surfaces at nongrazing angles [8]. We plan to investigate the variation of the truncated sea spectrum with respect to the wind speed and microwave frequency and to use the GMoM to compute the associated variation of the NRCS, both copolarized and cross-polarized. This will constitute a unique benchmark to test approximate scattering theories and to learn about their capability to modelize sea surface scattering on this part of the spectrum. We think in particular to higher order methods that predict cross-polarized scattering in the plane of incidence, and which validity and accuracy are difficult to assess.

\section{REFERENCES}

[1] F. Nathanson, J. Reilly, and M. Cohen, Radar Design Principles: Signal Processing and the Environment. Dules, VA, USA: SciTech, 1999.

[2] M. Long, Radar Reflectivity of Land and Sea, 3rd ed. Norwood, MA, USA: Artech House, 2001.

[3] V. Gregers-Hansen and R. Mital, "An empirical sea clutter model for low grazing angles," in Proc. IEEE Radar Conf., 2009, pp. 1-5.

[4] A. Voronovich and V. Zavorotny, "Theoretical model for scattering of radar signals in $K_{u}$ - and C-bands from a rough sea surface with breaking waves," Waves Random Media, vol. 11, no. 3, pp. 247-269, Jul. 2001.
[5] A. Mouche, B. Chapron, and N. Reul, "A simplified asymptotic theory for ocean surface electromagnetic wave scattering," Waves Random Complex Media, vol. 17, no. 3, pp. 321-341, Jun. 2007.

[6] G. Soriano and C. Guérin, "A cutoff invariant two-scale model in electromagnetic scattering from sea surfaces," IEEE Geosci. Remote Sens. Lett., vol. 5, no. 2, pp. 199-203, Apr. 2008.

[7] C.-A. Guérin, G. Soriano, and B. Chapron, "The weighted curvature approximation in scattering from sea surfaces," Waves Random Complex Media, vol. 20, no. 3, pp. 364-384, Jul. 2010.

[8] J. T. Johnson, "A numerical study of low-grazing-angle backscatter from ocean-like impedance surfaces with the canonical grid method," IEEE Trans. Antennas Propag., vol. 46, no. 1, pp. 114-120, Jan. 1998.

[9] G. Soriano and M. Saillard, "Modelization of the scattering of electromagnetic waves from the ocean surface," Progr. Electromagn. Res., vol. 10, no. 4, pp. 101-128, 2003.

[10] J. V. Toporkov, R. S. Awadallah, and G. S. Brown, "Issues related to the uses of a Gaussian-like incident field for low-grazing-angle scattering," $J$. Opt. Soc. Amer. A, Opt. Image Sci., vol. 16, no. 1, pp. 176-186, Jan. 1999.

[11] R. Chen and J. C. West, "Analysis of scattering from rough surfaces at large incidence angles using a periodic-surface moment method," IEEE Trans. Geosci. Remote Sens., vol. 33, no. 5, pp. 1206-1213, Sep. 1995.

[12] Z. Zhao, L. Li, J. Smith, and L. Carin, "Analysis of scattering from very large three-dimensional rough surfaces using MLFMM and raybased analyses," IEEE Antennas Propag. Mag., vol. 47, no. 3, pp. 20-30, Jun. 2005.

[13] P. Spiga, G. Soriano, and M. Saillard, "Scattering of electromagnetic waves from rough surfaces: A boundary integral method for low-grazing angles," IEEE Trans. Antennas Propag., vol. 56, no. 7, pp. 2043-2050, Jul. 2008.

[14] V. Tatarskii and M. Charnotskii, "Universal behaviour of scattering amplitudes for scattering from a plane in an average rough surface for small grazing angles," Waves Random Complex Media, vol. 8, no. 1, pp. 29-40, Jan. 1998.

[15] D. Colton and R. Kress, Integral Equations in Scattering Theory. NY, NY, USA: Wiley, 1983.

[16] P. A. Martin and P. Ola, "Boundary integral equations for the scattering of electromagnetic waves by a homogeneous dielectric obstacle," Proc. Roy. Soc. Edinburgh, A Math., vol. 123A, no. 1, pp. 185-208, Jan. 1993.

[17] G. Hsiao and R. Kleinman, "Mathematical foundations for error estimation in numerical solutions of integral equations in electromagnetics," IEEE Trans. Antennas Propag., vol. 45, no. 3, pp. 316-328, Mar. 1997.

[18] T. T. Ong, V. Celli, and A. M. Marvin, "General relation between surface impedance and surface curvature," J. Opt. Soc. Amer. A, Opt. Image Sci., vol. 11, no. 2, pp. 759-765, Feb. 1994.

[19] G. Soriano and M. Saillard, "Scattering of electromagnetic waves from two-dimensional rough surfaces with impedance approximation," J. Opt. Soc. Amer. A, Opt. Image Sci., vol. 18, no. 1, pp. 124-133, Jan. 2001.

[20] L. Tsang, J. A. Kong, K. H. Ding, and C. O. Ao, Scattering of Electromagnetic Waves: Numerical Simulations, J. A. Kong, Ed. Hoboken, NJ, USA: Wiley, 2001, ser. Wiley Series in Remote Sensing.

[21] S. Q. Li, C. H. Chan, M. Y. Xia, B. Zhang, and L. Tsang, "Multilevel expansion of the sparse-matrix canonical grid method for two-dimensional random rough surfaces," IEEE Trans. Antennas Propag., vol. 49, no. 11, pp. 1579-1589, Nov. 2001

[22] C. Tai, Generalized Vector and Dyadic Analysis: Applied Mathematics in Field Theory. Piscataway, NJ, USA: IEEE Press, 1997, ser. IEEE/OUP Series on Electromagnetic Wave Theory.

[23] L. A. Klein and C. T. Swift, "An improved model for the dielectric constant of sea water at microwave frequencies," IEEE Trans. Antennas Propag., vol. AP-25, no. 1, pp. 104-111, Jan. 1977.

[24] G. R. Valenzuela, "Depolarization of EM waves by slightly rough surfaces," IEEE Trans. Antennas Propag., vol. AP-15, no. 4, pp. 552-557, Jul. 1967.

[25] T. Elfouhaily, B. Chapron, K. Katsaros, and D. Vandemark, "A unified directional spectrum for long and short wind-driven waves," J. Geophys. Res., vol. 102, no. C7, pp. 15781-15 796, Jul. 1997.

[26] T. Elfouhaily and C. A. Guérin, "A critical survey of approximate scattering wave theories from random rough surfaces," Waves Random Media, vol. 14, no. 4, pp. R1-R40, Oct. 2004.

[27] P. Beckmann, "Scattering by non-Gaussian surfaces," IEEE Trans. Antennas Propag., vol. AP-21, no. 2, pp. 169-175, Mar. 1973.

[28] S. Peng and C. Wang, "Hardware accelerated MoM-PFFT method using graphics processing units," in Proc. IEEE Int. Symp. APSURSI, 2011, pp. 3152-3153.

[29] W. Yang, Z. Zhao, C. Qi, and Z. Nie, "Electromagnetic modeling of breaking waves at low grazing angles with adaptive higher order hierarchical Legendre basis functions," IEEE Trans. Geosci. Remote Sens., vol. 49, no. 1, pp. 346-352, Jan. 2011. 


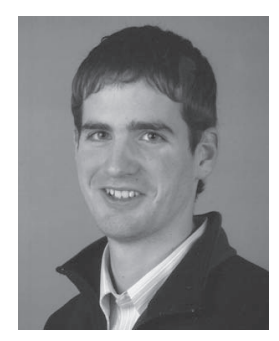

David Miret was born in Suresnes, France, in 1987. He received the M.S. degree in physics and engineering from the École Centrale Paris, Paris, France, in 2007. He is currently working toward the Ph.D. degree at DCNS, Toulon, France.

$\mathrm{He}$ is a Member of the DCNS Electromagnetic Compatibility Team collaborating with the Mediterranean Institute of Oceanography, Toulon, and the Fresnel Institute, Marseille, France.

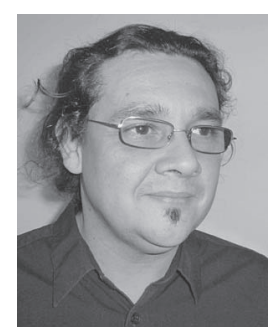

Gabriel Soriano was born in Paris, France, in 1972. He received the M.S. degree in physics and the Ph.D. degree in physics from the Paul Cezanne University, Marseille, France, in 1996 and 2000, respectively.

$\mathrm{He}$ is currently an Associate Professor with AixMarseille University, Marseille. He is a Member of the ElectroMagnetical and Optical Remote Sensing and CONCEPT research teams of the Fresnel Institute, Marseille. He works on electromagnetic wave scattering from rough surfaces and associated numedia on light polarization.

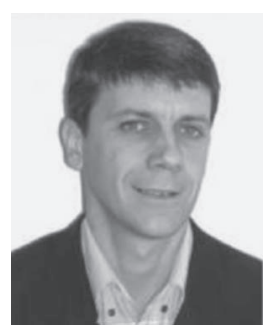

Marc Saillard was born in Marseille, France, in 1961. He received the "Agrégation" degree in physics and the Ph.D. degree from the University of Marseille, Marseille, in 1985 and 1990, respectively.

From 1990 to 1997, he was an Assistant Researcher with the Electromagnetic Optics Laboratory, National Center for Scientific Research, Marseille. In 1997, he was a Professor with the University of Marseille where he run the remote sensing group of Institut Fresnel, Marseille. In 2003, he moved to University of Toulon, La Garde, France, as a Professor, where he joined the remote sensing laboratory. In 2011, he was elected president of University of Toulon. His scientific interests lie in ocean microwave sensing, modelization and analysis of the sea surface radar signature, and imaging through random media. 